\title{
A population-based follow-up study shows high psychosis risk in women with PCOS
}

\author{
Salla Karjula ${ }^{1} \cdot$ Riikka K. Arffman ${ }^{1} \cdot$ Laure Morin-Papunen ${ }^{1} \cdot$ Stephen Franks ${ }^{2}$ - Marjo-Riitta Järvelin ${ }^{3,4}$. \\ Juha S. Tapanainen ${ }^{1,5} \cdot$ Jouko Miettunen $^{6,7} \cdot$ Terhi T. Piltonen $^{1}$
}

Received: 3 July 2021 / Accepted: 9 November 2021 / Published online: 29 November 2021

(c) The Author(s) 2021

\begin{abstract}
Polycystic ovary syndrome (PCOS) is a common endocrine disorder affecting up to $18 \%$ of women. Besides metabolic and fertility aspects, attention has lately been directed towards the detrimental effect of PCOS on psychological health. The objective of the study was to investigate whether women with PCOS are at higher risk for psychotic disorders. The study population derives from the Northern Finland Birth Cohort 1966 ( $N=5889$ women). The women with PCOS were identified by two simple questions on oligo-amenorrhea and hirsutism at age 31 . Women reporting both symptoms were considered PCOS $(N=124)$ and asymptomatic women as controls $(N=2145)$. The diagnosis of psychosis was traced using multiple national registers up to the year 2016. Symptoms of psychopathology were identified using validated questionnaires at age 31. Women with PCOS showed an increased risk for any psychosis by age 50 (HR [95\% CI] 2.99, [1.52-5.82]). Also, the risk for psychosis after age 31 was increased (HR 2.68 [1.21-5.92]). The results did not change after adjusting for parental history of psychosis, nor were they explained by body mass index or hyperandrogenism at adulthood. The scales of psychopathology differed between women with PCOS and non-PCOS controls showing more psychopathologies among the affected women. PCOS cases were found to be at a three-fold risk for psychosis, and they had increased psychopathological symptoms. PCOS should be taken into consideration when treating women in psychiatric care. More studies are required to further assess the relationship between PCOS and psychotic diseases.
\end{abstract}

Keywords Polycystic ovary syndrome $\cdot$ PCOS $\cdot$ Psychosis $\cdot$ Hirsutism $\cdot$ Testosterone

Terhi T. Piltonen

terhi.piltonen@oulu.fi

1 Department of Obstetrics and Gynecology, University Hospital of Oulu, University of Oulu, Medical Research Centre Oulu and PEDEGO Research Unit, Oulu, Kajaanintie 50, 90014 Oulu, Finland

2 Institute of Reproductive and Developmental Biology, Imperial College, London W12 ONN, UK

3 Department of Epidemiology and Biostatistics, Medical Research Council-PHE (Public Health England) Centre for Environment \& Health, School of Public Health, Imperial College, London W2 1PG, UK

4 Centre for Life Course Epidemiology, Faculty of Medicine, University of Oulu, FI-90014 Oulu, Finland

5 Department of Obstetrics and Gynecology, University of Helsinki and Helsinki University Hospital, FI-00014 Helsinki, Finland

6 Centre for Life Course Health Research, University of Oulu, Box 5000, FI-90014 Oulu, Finland

7 Medical Research Centre Oulu, Oulu University Hospital and University of Oulu, Oulu, Finland

\section{Introduction}

Polycystic ovary syndrome (PCOS) is the most common endocrine disorder of women, affecting up to $18 \%$ of reproductive-aged women (March et al. 2010). Unfortunately, this complex, chronic condition often remains undiagnosed and thus untreated (Piltonen et al. 2019; Teede et al. 2018). The diagnostic criteria of PCOS include ovulatory dysfunction/chronic anovulation, hyperandrogenism, and polycystic ovarian morphology; however, metabolic dysfunction and psychological illnesses are also dominant features of the syndrome.

Previous studies have shown that women with PCOS are at increased risk of psychological problems such as depression, anxiety, bipolar disorder, and disordered eating (Chen et al. 2020b, 2020c; Cooney et al. 2017; Karjula et al. 2017; Teede et al. 2018). The predisposing factors for these comorbidities remain unclear to date (Cooney et al. 2017; Teede et al. 2018). Even though genetic factors and family history 
of a psychiatric condition are the most common risk factors for psychological morbidities, various prenatal exposures and conditions during brain development, e.g., maternal obesity and stress, have also been shown to affect the probability of psychiatric morbidities later in life (Chen et al. 2020a; Kawai et al. 2004; Rice et al. 2007).

Psychosis and the specific diagnosis of schizophrenia represent the most severe forms of psychiatric disorders. Psychotic symptoms are characteristic of all psychotic syndromes, but the specific symptoms, duration, and effects on cognitive function vary (World Health Organization, 2018). Although several risk factors are known for psychosis, they explain only a minority of the cases. Indeed, several studies considering the etiology of psychosis show a multifactorial background with interaction between both genetic factors and the environment (Bearden \& Forsyth, 2018; Matheson et al 2011). A family history of psychosis is considered the most important risk factor, but some prenatal and neonatal factors, such as obstetric complications or viral infections, also increase the risk (Matheson et al. 2011).

While psychiatric and psychological disturbances are strongly associated with PCOS, not much is known about more severe psychiatric diseases, schizophrenia, and other psychoses. Two studies have concluded that women with PCOS are at higher risk of schizophrenia (Cesta et al. 2016; Chen et al., 2020c); however, psychotic disorders other than schizophrenia were not investigated. Given that only a few studies have suggested elevated scores for psychotic symptoms (Borghi et al. 2017; Elsenbruch et al. 2003), further research is warranted.

Recently, there has been increasing interest in defining the roles of multiple hormonal factors and metabolic alterations in the development of psychosis, of which many are also associated with PCOS. It has been shown-although inconsistently - that hyperandrogenism could be a risk factor for psychosis in women (Misiak et al. 2018). A hypothesis regarding the protective action of estrogen has also been presented (Huber et al. 2004; Riecher-Rössler 2017), which could explain the sex differences in schizophrenia, i.e., later onset, less severe illness, and somewhat lower prevalence in women (Jongsma et al. 2019; Novick et al. 2016; Van Der Werf et al. 2014). Higher prolactin (PRL) levels in drugnaïve first-episode psychosis female patients have also been reported (Delgado-Alvarado 2019; Riecher-Rössler et al. 2013). As for metabolic traits, patients diagnosed with psychosis, even before antipsychotic medication, often display dyslipidemia (Misiak et al. 2017), higher waist circumference (Cordes et al. 2017), and glucose intolerance (Perry et al. 2019). Additionally, hypotheses suggesting an inflammatory origin for the pathogenesis of schizophrenia and other psychoses have been proposed (Upthegrove et al. 2014).

Recent studies have linked psychological disturbances in women with PCOS to the early development of the syndrome, possibly through in utero androgen exposure (Risal et al. 2019; Stener-Victorin et al. 2019). Moreover, as obesity and related insulin resistance and high serum insulin levels aggravate hyperandrogenism and cause fetal stress, they may also increase the risk of transgenerational transmission of the syndrome and induce psychiatric morbidity in the offspring (Cesta et al. 2020; Hatanaka et al. 2017; Kong et al. 2018; Stener-Victorin et al. 2019). Interestingly, emerging data imply that the traits of PCOS, including psychiatric morbidity, can be carried over several generations (Cesta et al. 2020; Risal et al. 2019; Stener-Victorin et al. 2019).

Against this background, the objective of the present work was to investigate whether women with PCOS are at higher risk for psychotic disorders. Here, for the first time, the psychosis risk was assessed in a population-based studysetting identifying women with PCOS symptoms and linking the data with the national datasets. We also used symptomatic psychopathology scales to assess susceptibility to psychosis (Miettunen et al. 2011; Miettunen \& Jääskeläinen 2010). The study utilized the unique, longitudinal Northern Finland Birth Cohort dataset (NFBC1966), comprising all expected births in 1966 ( $N=5889$ women $)$ with a longitudinal follow-up to age 50 . We were also able to derive comprehensive national register data and a wide variety of possible confounding factors to investigate the risk of psychosis in affected women.

\section{Material and methods}

\section{Study population}

The NFBC1966 cohort population and validation of the characteristics related to PCOS diagnoses have been described previously (Karjula et al. 2017; Ollila et al. 2016; Taponen et al. 2003, 2004). In brief, the cohort includes individuals expected to be born in 1966 in Northern Finland ( $n$ total $=$ 12,$058 ; n$ female $=5889$ ), altogether comprising $96.3 \%$ of total births in the region (Rantakallio 1988, University of Oulu 1966). This study utilized the cohort data collected at the age of 31 years as well as the national register data of psychiatric diagnoses and medications. The characteristics of the study groups are presented in Table 1. Data were gathered via a questionnaire sent to all cohort subjects and from clinical examinations.

The questionnaire was sent to 5608 women of the cohort at age 31 (1996-1997), 4523 (81\%) of whom responded. The questionnaire included questions concerning clinical factors such as weight and height. PCOS was screened with the following questions: (1) Is your menstrual cycle longer than 35 days more than twice a year? (considered as having oligo-amenorrhea, OA); and (2) Do you have excessive, bothersome body hair? (considered as having hirsutism, $\mathrm{H}$ ). 
Table 1 Clinical and socioeconomic characteristics of the control women and women with PCOS at age 31 in the Northern Finland Birth Cohort, 1966

\begin{tabular}{|c|c|c|c|}
\hline Character & $\begin{array}{l}\text { Controls } \\
\text { Q1 } n=2145 \\
\text { Q2 } n=1376\end{array}$ & $\begin{array}{l}\text { PCOS } \\
n=124 \\
n=77\end{array}$ & $p$ Value \\
\hline $\mathrm{BMI}\left(\mathrm{kg} / \mathrm{m}^{2}\right)$ & $23.79(4.32)$ & $27.25(6.86)$ & $<0.001$ \\
\hline BMI & & & $<0.001$ \\
\hline$<25 \mathrm{~kg} / \mathrm{m}^{2}$ & $70.2 \%$ & $46.3 \%$ & \\
\hline$\geq 25 \mathrm{~kg} / \mathrm{m}^{2}$ & $21.6 \%$ & $28.1 \%$ & \\
\hline$\geq 30 \mathrm{~kg} / \mathrm{m}^{2}$ & $8.2 \%$ & $25.6 \%$ & \\
\hline Testosterone (nmol/l) & $0.98[0.75,1.61]$ & $1.39[1.17,1.79]$ & $<0.001$ \\
\hline $\begin{array}{l}\text { Testosterone (upper } \\
\text { quartile) }\end{array}$ & $21.8 \%$ & $57.8 \%$ & $<0.001$ \\
\hline FAI & $2.11[1.49,2.97]$ & $4.38[2.75,6.79]$ & $<0.001$ \\
\hline FAI (upper quartile) & $20.1 \%$ & $67.1 \%$ & $<0.001$ \\
\hline $\begin{array}{l}\text { Parental history psy- } \\
\text { chosis }\end{array}$ & $6.2 \%$ & $6.5 \%$ & 0.921 \\
\hline
\end{tabular}

Mean (standard deviation) or median with [25\% lower quartile and $75 \%$ upper quartile] or proportions (\%). The numbers of participants may vary due to lack of available data. $Q 1$ postal questionnaire, $Q 2$ clinical visit

In total, 463 women reported OA, 471 reported $\mathrm{H}$, and 153 reported having both symptoms (the latter category was considered to be women with PCOS). Women who were pregnant or using hormonal contraceptives or who did not permit the use of their personal register data were excluded from the final study population. The final analysis group at age 31 consisted of 2145 asymptomatic women (the control group), 325 women with isolated $\mathrm{OA}, 322$ with isolated $\mathrm{H}$, and 124 with PCOS. A flow chart of the study is presented in Fig. 1. Clinical examinations, including measurements of weight, height, serum testosterone (T), and free androgen index (FAI), were performed for 3127 women $(76 \%)$.

All participants provided informed consent, and the study was approved by the Ethics Committee of the Northern Ostrobothnia District in Finland (EETTMK 94/2011).

\section{Identification of psychoses}

For the identification of psychoses, several registers were utilized:

(1) Care Register for Health Care (CRHC) (1972-2016)

(2) Finnish outpatient registers: special health care (1998-2016) and primary health care (2011-2016)

(3) Social Insurance Institution (SII) registers: sick days (1974-1999), disability pensions (1974-2000), and reimbursable medicines (1974-2005)

(4) Finnish Centre for Pensions: disability pensions (1974-2016)

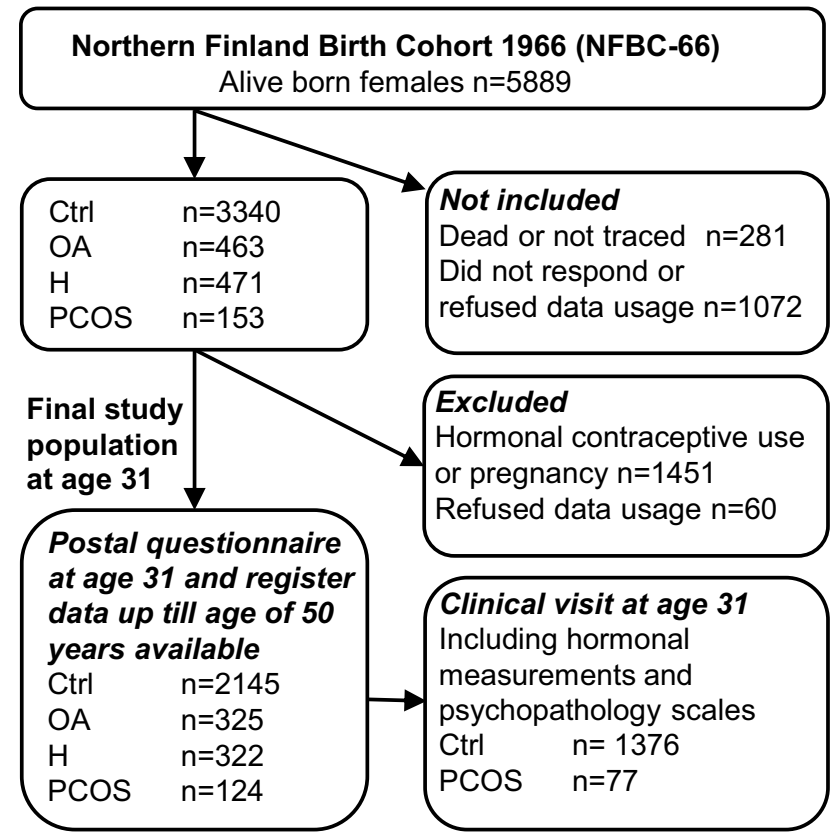

Ctrl, controls; OA, oligo-amenorrhea; $\mathrm{H}$, hirsutism; PCOS, polycystic ovary syndrome

Fig. 1 Flowchart of the study population

The identification of psychoses is described in more detail by Filatova et al. (2017). Diagnoses of non-organic psychosis were included up to 2016 (i.e., ICD-8 295-299; ICD-9 295, 2961E, 2962E, 2963E, 2964E, 2967, 297-299, 2988A, 2989X; ICD-10 F20, F22-F25, F28-F29, F302, F312, F315, F323, F333), representing the any psychosis group (Supplementary Table 1). For subgroup analysis, we separated the psychosis cases into schizophrenia and other psychosis as shown in Supplementary Table 1. For estimating the risk of psychosis between ages 31 and 50, we used the first onset of diagnoses of psychoses from age 31 onward.

\section{Parental psychosis}

Parental psychosis was defined as a parent (mother and/or father) having non-organic psychosis (i.e., ICD-8 295-299; ICD-9 295, 2961E, 2962E, 2963E, 2964E, 2967, 297-299; ICD-10 F20, F22-F29) at any time between 1964 and 2005. Information about parental psychosis was available from the disability pension register of the Finnish Centre for Pensions (1964-2005) and the CRHC (1972-2005), including outpatient registers from special health care (1998-2005). The proportions of parental psychosis in different study groups are shown in Table 1. There was no difference in the proportions of parental history of psychoses between the PCOS group $(6.5 \%)$ and the controls $(6.2 \% ; p=0.921)$. 


\section{Psychopathology scales}

As part of the clinical visit, the participants filled out a questionnaire consisting of mental health-related true/false (scored 0/1) questions collected from several psychological scales. The scales are used for the identification of psychopathological symptoms in individuals (Miettunen et al. 2011). The psychopathology scales used were the Social Anhedonia Scale (SAS), Physical Anhedonia Scale (PHAS), Perceptual Aberration Scale (PAS), Hypomanic Personality Scale (HPS), Bipolar II scale (BIP2), and Schizoidia Scale (SCHD). A more detailed description of the scales utilized in the same birth cohort has been published (Miettunen et al. 2011; Miettunen \& Jääskeläinen 2010). In cases where more than $10 \%$ of the response items were missing, the scale was excluded.

\section{Confounding variables}

\section{Body mass index}

In the clinical examinations, weight $(\mathrm{kg})$ was measured on a digital scale which was calibrated regularly. Height $(\mathrm{cm})$ was measured twice, using a standard and calibrated stadiometer. Body mass index (BMI) was calculated in $\mathrm{kg} / \mathrm{m}^{2}$. If a measurement was missing, the self-reported values were used. No statistical difference was observed between the measured and the self-reported BMIs (Ollila et al. 2016).

\section{Testosterone and FAI}

Serum $\mathrm{T}$ and the sex hormone binding globulin (SHBG) were assayed at age 31 , as previously described (Karjula et al. 2017). Testosterone levels were assayed using Agilent triple quadrupole $6410 \mathrm{LC} / \mathrm{MS}$ equipment with an electrospray ionization source, operating in positive-ion mode (Agilent Technologies). At age 31, SHBG was assayed using fluoroimmunoassay (Wallac, Inc. Ltd., Turku, Finland). FAI was calculated as follows: 100xT (nmol/L)/SHBG (nmol/L).

\section{Socioeconomic status/education}

Socioeconomic status was based on years of education. The variable was classified into three groups according to the number of education years: $\leq 9,9-12$, and $>12$ years.

\section{Statistical analysis}

Statistical analyses were performed using SPSS version 25 for Windows. The analysis of categorical variables was carried out using the Chi-square test and Fisher's exact test when appropriate. Cox regression analysis (using hazard ratios, HR) was used to estimate the association between
PCOS and psychotic disorders. Kaplan-Meier survival analysis (Mantel-Cox estimation) was used to estimate the incidence of psychotic disorders. Parental history of psychosis, BMI, education, testosterone, or FAI was used as a covariate. The analyses of psychometric scales were done using Student's $t$-test for variables with normal distributions and the Mann-Whitney $U$-test as a non-parametric test. The data was also analyzed after removing women with a diagnosis of psychosis before age 31 to assess the incidence after the establishment of PCOS symptoms. The results are reported as means with standard deviations (SD), medians (25\% and $75 \%$ quartiles), prevalence (\%), and HR with a $95 \%$ confidence interval (CI). $p$ Values $<0.05$ were considered statistically significant.

\section{Results}

\section{Lifetime prevalence and incidence of psychosis}

The lifetime prevalence (until age 50) of any psychoses in women with PCOS was $8.1 \%$ (compared to $2.8 \%$ for controls; $p=0.004$ ). The prevalence of schizophrenia was not significantly higher in the PCOS group $(2.4 \%$ vs. $1.2 \% ; p$ $=0.245)$, but the number of cases was severely limited. However, the lifetime prevalence for psychoses other than schizophrenia was increased in PCOS: $5.6 \%$ vs. $1.6 \%$ ( $p=$ 0.006) (Fig. 2A).

When considering the diagnoses of psychosis from age 31 to 50, the incidence of any psychosis in PCOS women was $5.8 \%$, compared to $2.2 \%$ for controls ( $p=0.023$ ). There were no differences in diagnoses of schizophrenia between the PCOS group $(0.8 \%)$ and controls $(0.9 \% ; p=0.96)$ during this period, whereas the incidence of other psychoses was $5.7 \%$ (compared to $1.5 \%$ for controls; $p=0.004$ ) (Fig. 2B). No differences were found between women with isolated OA or $\mathrm{H}$ compared to controls; thus, these two groups were not included in further analyses (Fig. 2A,B).

\section{The risks of lifetime diagnoses of psychosis until age 50}

In the Cox regression model (Fig. 3), the risk of any psychosis until age 50 in women with PCOS symptoms was increased (HR 2.99, 95\% confidence interval [95\% CI] 1.53-5.83). After adjusting for parental psychoses, the risk remained high (HR 2.98, 95\% CI 1.52-5.82). The risk of schizophrenia did not reach statistical significance (HR 2.04, 95\% CI 0.62-6.74), likely due to the low number of cases in the PCOS group. In contrast, the risk of other psychoses was higher in women with PCOS (HR 3.66, 95\% CI 1.62-8.26), and adjusting for parental history of psychosis did not affect the result (HR 3.62, 95\% CI1.61-6.10) even though it was 


\section{A}

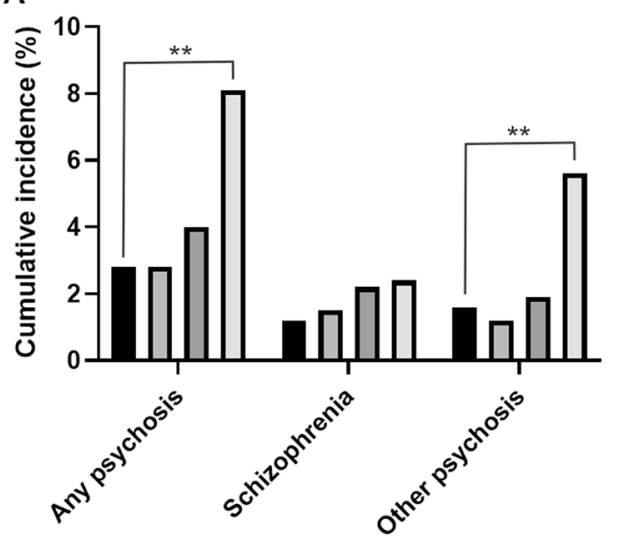

B

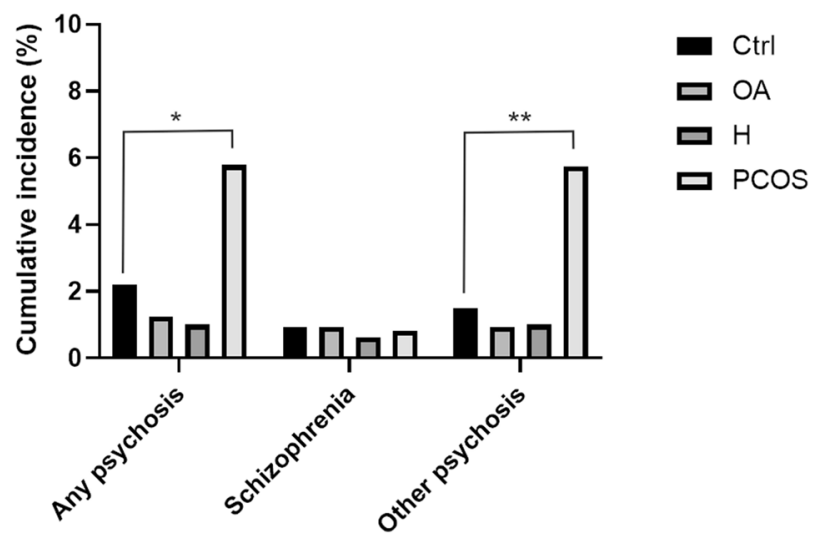

Fig. 2 Cumulative incidence of psychosis $\mathbf{a}$ until age 50 and $\mathbf{b}$ from age 31 to 50 years, in women with isolated oligo-amenorrhea (OA), women with isolated hirsutism $(\mathrm{H})$, and women with PCOS $(\mathrm{OA}+\mathrm{H})$ compared to controls (Ctrl) $(* p<0.05, * * p<0.01$, *** $p<0.001)$

still strongly associated with the risk of all psychoses (data not shown).

\section{The risks of psychoses from age $\mathbf{3 1}$ to age $\mathbf{5 0}$}

The Cox regression analysis showed that the risk of having any psychoses from age 31 to age 50 was elevated significantly in women with PCOS (unadjusted HR 2.69, 95\% CI 1.21-5.94) and this result did not change even after adjusting for parental history of psychoses (adj. HR 2.68, 95\% CI 1.21-5.92). Furthermore, after adjusting for BMI and education, the risks were not reduced (Table 2 ).

\section{The effect of hyperandrogenism on the risk of psychoses}

Of the women with PCOS who attended the clinical examination and blood tests at the age of $31,9.5 \%$ received new diagnoses of psychosis between ages 31 and 50, compared (a)

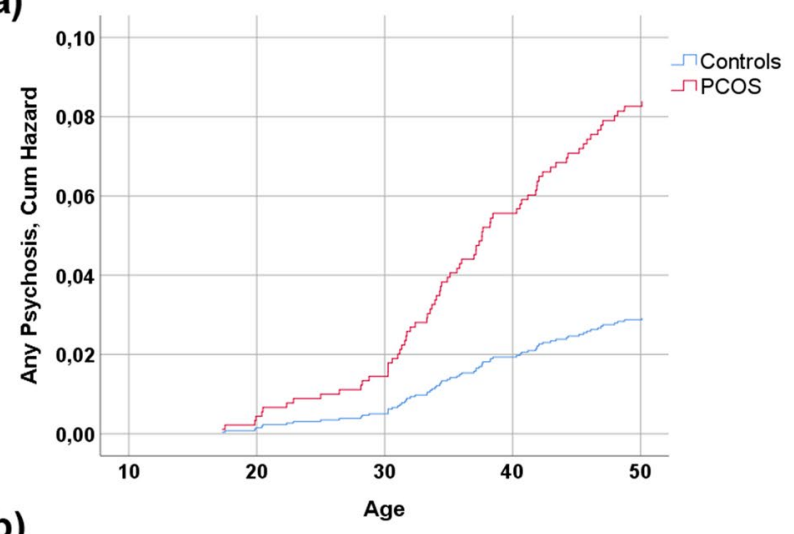

(b)

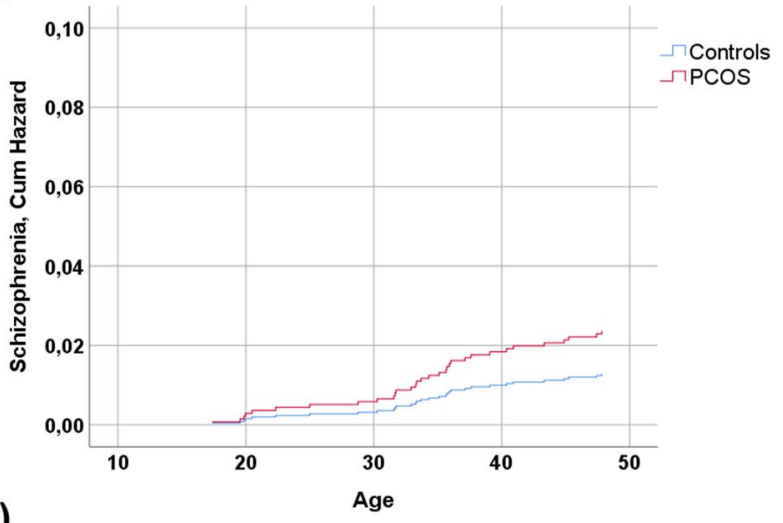

(c)

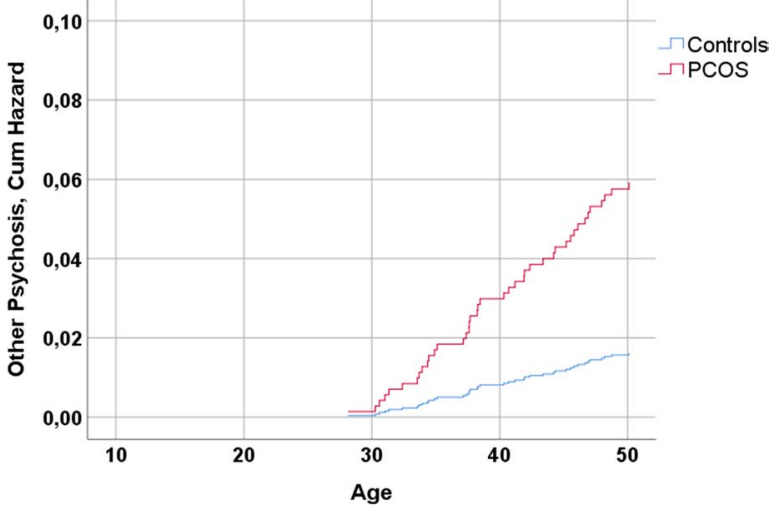

Fig. 3 Cumulative hazard functions of age at onset of psychosis in women with PCOS and non-PCOS controls: a any psychosis, $\mathbf{b}$ schizophrenia, and $\mathbf{c}$ other psychosis up to age 50. The figures also include hazard ratios (HR) and their 95\% confidence intervals from Cox regression analysis for a psychoses, $\mathbf{b}$ schizophrenia, and $\mathbf{c}$ other psychosis. The data has been adjusted for parental history of psychosis. On the $\mathrm{Y}$ axis, the incidence is presented as part of the whole, where 1.0 represents the whole study population

to $2.8 \%$ for the controls (unadjusted HR 3.58, $95 \%$ CI 1.60-8.04). When adjusting for testosterone (at age 31), the risk was slightly reduced (HR 3.03, 95\% CI 1.26-7.31), as was the case also when adjusting for FAI (HR 2.85, 95\% CI 1.09-7.49) (Table 3). However, neither serum testosterone 
Table 2 The risk of any psychoses, schizophrenia, and other psychosis in women with PCOS between ages 31 and 50 in the Cox regression model. The risk is presented in the form of hazard ratios

\begin{tabular}{llll}
\hline & Any psychosis & Schizophrenia & Other psychosis \\
PCOS $n=121-122 *$ & $\begin{array}{l}n=7(5.8 \%) \\
n=47(2.3 \%)\end{array}$ & $\begin{array}{l}n=1(0.8 \%) \\
n=20(0.9 \%)\end{array}$ & $\begin{array}{l}n=7(5.7 \%) \\
n=32(1.5 \%)\end{array}$ \\
Ctrl $n=2132-2139 *$ & $2.69(1.21-5.94)$ & $0.89(0.12-6.62)$ & $3.95(1.74-8.95)$ \\
\hline Unadjusted (HR [95\% CI]) & $2.81(1.24-6.37)$ & $0.89(0.12-6.78)$ & $4.16(1.78-9.74)$ \\
Adj. BMI at age 31 & $2.68(1.21-5.92)$ & $0.89(0.12-6.59)$ & $3.95(1.74-8.94)$ \\
Adj. parental history of psychosis & $2.64(1.19-5.85)$ & $0.87(0.12-6.46)$ & $3.87(1.71-8.80)$ \\
Adj. education &
\end{tabular}

$H R$ hazard ratio, $C I$ confidence interval, $B M I$ body mass index. *Numbers vary due to missing data from some individuals for specific variables
Table 3 The risk of any psychosis, schizophrenia, and other psychosis in women with PCOS between ages 31 and 50 in a Cox regression model adjusted with BMI, testosterone, and free androgen index
(FAI). The data includes only women attending clinical examination at age 31 and for whom testosterone and sex hormone binding globulin (SHBG) measurements are thus available

\begin{tabular}{llll}
\hline & Any psychosis & Schizophrenia & Other psychosis \\
PCOS $n=74-75^{*}$ & $n=7(9.5 \%)$ & $n=1(1.3 \%)$ & $n=7(9.3 \%)$ \\
Ctrl $n=1368-1372 *$ & $n=37(2.8 \%)$ & $n=14(1.0 \%)$ & $n=26(1.9 \%)$ \\
\hline Unadjusted [HR (95\%CI)] & $3.58(1.60-8.04)$ & $1.32(0.17-10.06)$ & $5.08(2.21-11.70)$ \\
Adj. BMI at age 31 & $3.94(1.72-9.04)$ & $1.53(0.20-11.89)$ & $5.34(2.25-12.68)$ \\
Adj. testosterone & $3.03(1.26-7.31)$ & $0.98(0.12-8.29)$ & $4.53(1.82-11.32)$ \\
Adj. FAI & $2.85(1.09-7.49)$ & $1.52(0.17-13.72)$ & $3.87(1.41-10.59)$ \\
\hline
\end{tabular}

$H R$ hazard ratio, $C I$ confidence interval, $B M I$ body mass index, $F A I$ free androgen index. *Numbers vary due to missing data from some individuals for specific variables

nor FAI was independently associated with the risk of psychosis (data not shown).

\section{The results of psychopathology scales}

For women with PCOS symptoms, the scales for PAS were significantly higher than for controls $(p=0.024)$. The mean (SD) HPS score for the PCOS group was 14.88 (8.59), compared to 11.61 (6.97) in controls $(p=0.002)$, and for the SCHD, it was 3.14 (1.49) in PCOS vs. 2.77 (1.38) in controls. No statistically significant differences were found between PCOS cases and controls in SAS, PHAS, or BIP2 (Table 4).

\section{Discussion}

This community-based population study shows for the first time that women with PCOS are at a three-fold increased risk of psychoses compared to non-PCOS controls, at least until age 50. Indeed, the fact that the lifetime prevalence of psychosis was $8.1 \%$ in PCOS subjects compared to $2.8 \%$ in controls warrants attention. An elevated risk of psychoses was also detected during the follow-up period started after the establishment of the PCOS diagnosis (ages 31-50). The results were independent of parental history of psychosis,
Table 4 Psychopathology scales in control women and in women with PCOS at age 31 without previous diagnosis of psychosis

\begin{tabular}{llll}
\hline Scale & $\begin{array}{l}\text { Controls } \\
n=1277-1281^{*} \\
\text { median [IQR] or mean (SD) }\end{array}$ & $\begin{array}{l}\text { PCOS } \\
n=71-72 * \\
\text { median [IQR] or } \\
\text { mean (SD) }\end{array}$ & $p$ Value \\
\hline SAS & $7.0[5.0,10.0]$ & $8.0[6.0,11.0]$ & 0.065 \\
PHAS & $12.00[9.0,16.0]$ & $13.0[9.0,16.0]$ & 0.330 \\
PAS & $1.0[0,3.0]$ & $2.0[1.0,4.0]$ & 0.024 \\
HPS & $11.61(6.97)$ & $14.88(8.59)$ & 0.002 \\
BIP2 & $10.32(3.68)$ & $11.29(4.77)$ & 0.093 \\
SCHD & $2.77(1.38)$ & $3.14(1.49)$ & 0.024 \\
\hline
\end{tabular}

The results are reported as mean (standard deviation) or median with [25\% lower quartile and $75 \%$ upper quartile] when appropriate. The differences are analyzed using Student's t-test or Mann-Whitney $U$-test. The psychopathology scales used were the Social Anhedonia Scale (SAS), Physical Anhedonia Scale (PHAS), Perceptual Aberration Scale (PAS), Hypomanic Personality Scale (HPS), Bipolar II scale (BIP2), and Schizoidia Scale (SCHD). *Numbers vary due to missing data from some individuals for specific variables

the most strongly predisposing factor for severe mental illness (Matheson et al. 2011). The results also indicated that isolated PCOS symptoms (H or OA alone), BMI, and biochemical hyperandrogenism in adulthood were not major contributors to psychosis risk in women with PCOS. 
Recently, there has been increasing interest in psychological distress in women with PCOS. Affected women have been found to have an increased risk of anxiety, depression, bipolar disorder, and eating disorders compared to non-PCOS controls (Cesta et al. 2016; Chen et al., 2020b, 2020c; Cooney et al. 2017; Karjula et al. 2017; Teede et al. 2018). However, previous evidence for the risk of severe mental disorders in affected women is limited, highlighting the need to explore this area. Interestingly, a previous study showed that women at their first admissions due to schizophrenia more often showed PCOS-related symptoms (such as pre-existing menstrual disturbances, hair loss, hirsutism, and infertility) than age-matched healthy women (Riecher-Rössler 2002). A previous study by Cesta et al., also performed on a Nordic population, did not assess the risk of other psychoses in PCOS patients; however, they did report an increased risk of schizophrenia, in contrast to our results (Cesta et al. 2016). In addition, a recent Taiwanese cohort study reported a greater than six-fold risk of schizophrenia in PCOS subjects (Chen et al. 2020c). For our study, the number of PCOS cases with schizophrenia was likely resulting in lack of power for analysis, and thus no reliable conclusions can be drawn regarding the association of schizophrenia and PCOS. However, in line with our data, another Taiwanese population-based cohort study found the HR for schizophrenia to be nonsignificant during a 10-year followup period (HR 0.84, 95\% CI 0.43-1.59) (Hung et al. 2014).

The results of the psychopathology scale analysis, including PAS, HPS, and SCHD, indicated higher psychopathology in women with PCOS. These psychopathology scales are known to be associated with psychotic diseases and/ or symptoms (Miettunen et al. 2011), thus supporting the notion of increased risk of psychosis in women with PCOS. Previously, two studies investigated psychological disturbances using the symptom checklist-90 (SCL-90) scales and reported increased psychoticism scores in women with PCOS (Borghi et al. 2017; Elsenbruch et al. 2003), in line with our results.

With increasing evidence of psychological distress in PCOS, the number of studies on the mechanisms behind the morbidity is also increasing. These studies consider the factors associated with the syndrome, such as hyperandrogenism, obesity, and insulin resistance (Cooney et al. 2017; Greenwood et al. 2019). Other recent studies considering the predisposing factors for psychotic disorders and other psychiatric diseases have focused on hormonal and metabolic factors (Misiak et al., 2017, 2018; Perry et al., 2019).

It has been reported that patients with severe mental disorders are more commonly obese, possibly due to antipsychotic medication as well as an unhealthy lifestyle (De Hert et al., 2011). In the present study, the risk of psychosis did not decrease after adjusting for BMI at age 31 . In line with our finding, higher BMI was poorly correlated to psychosis risk in a previous study (Shah et al. 2019). Considering psychological distress in PCOS subjects, the syndrome itself has been shown to be an independent risk factor, although a high BMI might serve as a predisposing factor for depression and anxiety in the affected women (Cooney et al. 2017; Greenwood et al. 2019). The fact that weight gain aggravates PCOS symptoms while also increasing its prevalence supports the link between PCOS and psychological distress (Teede et al. 2013, 2018). Insulin resistance also appears to play a role in the psychological morbidity in PCOS, and in support of this, metformin therapy has been shown to reduce risk for bipolar disorder in this population (Chen 2020a).

Previous studies have implied a protective role for estrogen regarding psychosis risk (Huber et al.2004; Anita Riecher-Rössler 2017), while higher and rising estradiol levels during the menstrual cycle have been shown to relieve psychotic episodes and symptoms (Bergemann et al. 2007). Furthermore, physiological hypoestrogenism related to menopause also seems to be associated with an increased risk of schizophrenia (Searles et al. 2018). Theoretically, the link between low estrogen levels and psychosis risk may also relate to anovulation and a lack of high estrogen peaks in PCOS subjects due to absent menstrual cycles.

In the present study, the risk of psychosis in women with PCOS was slightly decreased after adjusting for T or FAI; however, in the whole population, high serum androgen levels in adulthood did not present as an independent risk factor (data not shown). Moreover, isolated PCOS symptoms (H or OA) were not associated with the risk of psychosis. This could underline the specific role of early exposure to hyperandrogenism in women with PCOS, as systemic hyperandrogenism in adulthood does not seem to present as a high-risk factor for psychosis in the general female population (Misiak et al., 2018). Indeed, exposure to hyperandrogenism in utero appears to provoke mental disturbances, in addition to the PCOS phenotype, in animal models (Stener-Victorin et al., 2019). On the other hand, high testosterone levels in the perinatal period promote abnormal synaptic development and sexual dimorphism in the brain, providing mechanisms for an increased risk of neurocognitive impairments such as ADHD and autism (Auyeung et al. 2013; Hatanaka et al. 2017). This could also provide the link between PCOS and psychiatric comorbidity related to the syndrome.

Given that family history is the most important factor for psychosis risk, transgenerational transmission (TT) should also be considered. In PCOS, TT and related hyperandrogenism are evidenced by recent studies following the offspring of PCOS-like mice to the third generation, supported by human epidemiological data (Mimouni et al. 2021; Risal et al. 2019). As for inherited traits of psychological morbidity, a recent study of register data showed that the daughters of mothers with PCOS presented more 
often with male-prominent neuropsychiatric diseases-i.e., autism spectrum disorders and ADHD (Cesta et al., 2020). Whether this also applies to more severe mental illnesses, such as psychosis, remains to be investigated.

There are strengths but also some limitations in the present study. The strengths include the population-based, longitudinal approach. Moreover, this prospectively collected data was also linked to the comprehensive national register data regarding psychiatric diagnoses. The Finnish national registers have been shown to be good and reliable data sources (Perälä et al., 2007). An extensive list of confounding factors was also available, as well as the history of parental psychosis. The fact that we were able to identify a female population with a high risk of developing psychiatric disease using only two simple questions about menstrual cycles and excessive hair growth is worth noting. The self-reported diagnosis of PCOS might still be considered a limitation, even though a hormonal, metabolic, and psychological profile typical for women with PCOS has been described extensively in our previous studies with this population (Karjula et al. 2017; Ollila et al. 2016; Taponen et al. 2003, 2004). Given that psychoses are highly healthburdening conditions, the related possible low attendance to cohort data collection could affect the result as the PCOS diagnosis would not have been established for these individuals (Haapea et al., 2008). Additionally, as existing psychosis and antipsychotic medication may cause excessive weight gain and menstrual irregularities, thus increasing the probability of PCOS symptoms, we conducted an analysis of psychosis incidence after the establishment of PCOS diagnosis at the age of 31 (De Hert et al., 2011; Gleeson et al. 2016; Teede et al. 2013, 2018). However, this age point may be relatively late to assess schizophrenia as the disease is commonly diagnosed in one's twenties (Van Der Werf et al. 2014). One of the major limitations was the low number of schizophrenia and psychosis cases among the PCOS population. For schizophrenia, the high confidential interval for risk implies that there was not enough power for the analysis to detect the difference in the risk. Given all mentioned before, this may have led to underestimation of the prevalence of severe psychiatric comorbidity in women with PCOS. This underlines the need for further studies with bigger study populations. Furthermore, the current population-based sample was unselected, but genetically homogeneous and the sample consisted mainly of women with white Caucasian background. Based on this, the results may not be generalizable to more heterogeneous populations.

\section{Conclusions}

The fact that women with PCOS seem to have a high risk of psychiatric comorbidities warrants devoting more resources and greater efforts to improve awareness, treatment modalities, and quality of life (Piltonen et al. 2019; Teede et al. 2018). Especially as the physical health of patients with severe mental illnesses has been shown to be poorly examined and treated (De Hert et al. 2011; Lambert et al. 2018), guidelines and recommendations for treating somatic diseases in women with severe mental illnesses are needed.

Based on the present results, the association of severe mental disorders and PCOS should be acknowledged in clinical practice. Future research should focus on investigating the mechanisms behind this association, thus enabling prevention and new treatment strategies.

Supplementary Information The online version contains supplementary material available at https://doi.org/10.1007/s00737-021-01195-4.

Author contribution S.K., T.T.P., and J.M. designed the study. S.K. conducted analyses. S.K., T.T.P., and R.K.A. interpreted the data and wrote the manuscript. M.R.J. participated in data collection as a scientific director of the NFBC. L.M.P., S.F., J.T., M.R.J., and J.K. gave clinical advice and revised the manuscript. All authors approved the final version.

Funding Open Access funding provided by University of Oulu including Oulu University Hospital. The study was funded by the Finnish Medical Foundation, the Northern Ostrobothnia Regional Fund, the University of Oulu Scholarship Foundation, Emil Aaltonen Foundation, the Academy of Finland (104781, 120315, 129269, 268336, 321763, $315921,1114194,24300796$ ), the Sigrid Juselius Foundation, Biocenter Oulu, University Hospital Oulu and University of Oulu (75617), Medical Research Center Oulu, National Institute for Health Research (UK), Genesis Research Trust (UK), and Medical Research Council, UK (G0500539, G0600705, G1002319, G0802782).

Data availability NFBC data is available from the University of Oulu, Infrastructure for Population Studies. Permission to use the data can be applied for research purposes via electronic material request portal. In the use of data, we follow the EU general data protection regulation (679/2016) and Finnish Data Protection Act. The use of personal data is based on cohort participant's written informed consent at his/her latest follow-up study, which may cause limitations to its use. Please, contact NFBC project center (NFBCprojectcenter@oulu.fi) and visit the cohort website (www.oulu.fi/nfbc) for more information.

\section{Declarations}

Conflict of interest The authors declare no competing interests.

Ethics approval The study was approved by the Ethics Committee of the Northern Ostrobothnia Hospital District in Finland (EETTMK 94/2011), and it was performed in accordance of the Declaration of Helsinki.

Informed consent All participants provided written informed consent for participation, use of the collected data, and publication. 
Open Access This article is licensed under a Creative Commons Attribution 4.0 International License, which permits use, sharing, adaptation, distribution and reproduction in any medium or format, as long as you give appropriate credit to the original author(s) and the source, provide a link to the Creative Commons licence, and indicate if changes were made. The images or other third party material in this article are included in the article's Creative Commons licence, unless indicated otherwise in a credit line to the material. If material is not included in the article's Creative Commons licence and your intended use is not permitted by statutory regulation or exceeds the permitted use, you will need to obtain permission directly from the copyright holder. To view a copy of this licence, visit http://creativecommons.org/licenses/by/4.0/.

\section{References}

B Auyeung MV Lombardo S Baron-Cohen 2013 Prenatal and postnatal hormone effects on the human brain and cognition Pflugers Arch 4655557571 https://doi.org/10.1007/s00424-013-1268-2

Bearden CE., \& Forsyth JK. (2018). The many roads to psychosis: recent advances in understanding risk and mechanisms. Dec 3;7:F1000Research. https://doi.org/10.12688/f1000research. 16574.1

N Bergemann P Parzer B Runnebaum F Resch C Mundt 2007 Estrogen, menstrual cycle phases, and psychopathology in women suffering from schizophrenia Psychol Med 371014271436 https://doi.org/ 10.1017/S0033291707000578

L Borghi D Leone E Vegni V Galiano C Lepadatu P Sulpizio E Garzia 2017 Psychological distress, anger and quality of life in polycystic ovary syndrome: associations with biochemical, phenotypical andsocio-demographic factors J Psychosom Obstet Gynecol 392 $128137 \mathrm{https} / / /$ doi.org/10.1080/0167482X.2017.1311319

CE Cesta M Månsson C Palm P Lichtenstein AN Iliadou M Landén 2016 Polycystic ovary syndrome and psychiatric disorders: comorbidity and heritability in a nationwide Swedish cohort Psychoneuroendocrinology 73196203 https://doi.org/10.1016/j. psyneuen.2016.08.005

CE Cesta AS Öberg A Ibrahimson I Yusuf H Larsson C Almqvist BM D’Onofrio CM Bulik L Fernández De La Cruz D Mataix-Cols M Landén MA Rosenqvist 2020 Maternal polycystic ovary syndrome and risk of neuropsychiatric disorders in offspring: prenatal androgen exposure or genetic confounding? Psychological Medicine. 50 4616624 https://doi.org/10.1017/S0033291719000424

X Chen L Kong TT Piltonen M Gissler H Larsson C Lavebratt 2020a Association of polycystic ovary syndrome or anovulatory infertility with offspring psychiatric and mild neurodevelopmental disorders: a Finnish population-based cohort study Hum Reprod 3510 23362347 https://doi.org/10.1093/humrep/deaa192

SF Chen YC Yang CY Hsu YC Shen 2020b Risk of bipolar disorder in patients with polycystic ovary syndrome: A nationwide population-based cohort study J Affect Disord 263458462 https://doi. org/10.1016/j.jad.2019.12.007

Chen SF, Yang YC, Hsu CY, Shen YC (2020c) Risk of schizophrenia in patients with polycystic ovary syndrome: a nationwide populationbased cohort study from Taiwan. J Psychosom Obstet Gynecol 0(0):1-7. https://doi.org/10.1080/0167482X.2020.1735342

LG Cooney I Lee MD Sammel A Dokras 2017 High prevalence of moderate and severe depressive and anxiety symptoms in polycystic ovary syndrome: a systematic review and meta-analysis Hum Reprod 32510751091 https://doi.org/10.1093/humrep/dex044

Cordes J, Bechdolf A, Engelke C, Kahl KG, Balijepalli C, Lösch C, Klosterkötter J, Wagner M, Maier W, Heinz A, de Millas W, Gaebel W, Winterer G, Janssen B, Schmidt-Kraepelin C, Schneider F, Lambert M, Juckel G, Wobrock T, ... Moebus S. (2017). Prevalence of metabolic syndrome in female and male patients at risk of psychosis. Schizophrenia Research. 181:38-42. https://doi.org/ 10.1016/j.schres.2016.09.012

Delgado-Alvarado M, Tordesillas-Gutierrez D, Ayesa-Arriola R, Canal M, de la Foz VO, Labad J, Crespo-Facorro B (2019) Plasma prolactin levels are associated with the severity of illness in drug-naive first-episode psychosis female patients. Arch Womens Ment Health 22(3):367-373. https://doi.org/10.1007/ s00737-018-0899-x

M Hert De D Cohen J Bobes M Cetkovich-Bakmas S Leucht DM Ndetei JW Newcomer R Uwakwe I Asai HJ Möller S Gautam J Detraux CU Correll 2011 Physical illness in patients with severe mental disorders. II. Barriers to care, monitoring and treatment guidelines, plus recommendations at the system and individual level World Psychiatry. 10213851 https://doi.org/10.1002/j. 2051-5545.2011.tb00036.x

S Elsenbruch S Hahn D Kowalsky AH Öffner M Schedlowski K Mann OE Janssen 2003 Quality of life, psychosocial well-being, and sexual satisfaction in women with polycystic ovary syndrome $\mathrm{J}$ Clin Endocrinol Metab 881258015807 https://doi.org/10.1210/ jc.2003-030562

S Filatova R Marttila H Koivumaa-Honkanen T Nordström J Veijola P Mäki GM Khandaker M Isohanni E Jääskeläinen K Moilanen J Miettunen 2017 A comparison of the cumulative incidence and early risk factors for psychotic disorder in young adults in the Northern Finland Birth Cohorts 1966 and 1986 Epidemiology and Psychiatric Sciences. 263314324 https://doi.org/10.1017/ S2045796016000123

PC Gleeson R Worsley E Gavrilidis S Nathoo Ng., Lee S, \& Kulkarni J. 2016 Menstrual cycle characteristics in women with persistent schizophrenia Aust N Z J Psychiatry 505481 487 https://doi.org/10.1177/0004867415590459

EA Greenwood LA Pasch K Shinkai MI Cedars HG Huddleston 2019 Clinical course of depression symptoms and predictors of enduring depression risk in women with polycystic ovary syndrome: results of a longitudinal study Fertil Steril 1111147 156 https://doi.org/10.1016/j.fertnstert.2018.10.004

M Haapea J Miettunen MK Isohanni JM Veijola E Läärä MR Järvelin MI Joukamaa 2008 Non-participation in a field survey with respect to psychiatric disorders Scandinavian Journal of Public Health. 367728736 https://doi.org/10.1177/1403494808092250

Y Hatanaka T Kabuta K Wada 2017 Disturbance in maternal environment leads to abnormal synaptic instability during neuronal circuitry development In Frontiers in Neuroscience. 61135 https:// doi.org/10.3389/fnins.2017.00035

TJ Huber M Borsutzky U Schneider HM Emrich 2004 Psychotic disorders and gonadal function: evidence supporting the oestrogen hypothesis Acta Psychiatr Scand 1094269274 https://doi.org/ 10.1046/j.1600-0447.2003.00251.x

JH Hung LY Hu SJ Tsai AC Yang MW Huang PM Chen SL Wang T Lu CC Shen 2014 Risk of psychiatric disorders following polycystic ovary syndrome: a nationwide population-based cohort study PLoS ONE. 95 e97041 https://doi.org/10.1371/journal. pone.0097041

HE Jongsma C Turner JB Kirkbride PB Jones 2019 International incidence of psychotic disorders, 2002-17: a systematic review and meta-analysis The Lancet Public Health. 45 e229 e244 https:// doi.org/10.1016/S2468-2667(19)30056-8

S Karjula L Morin-Papunen J Auvinen A Ruokonen K Puukka S Franks M-R Järvelin JS Tapanainen J Jokelainen J Miettunen TT Piltonen 2017 Psychological distress is more prevalent in fertile age and premenopausal women with pcos symptoms: 15-year follow-up Journal of Clinical Endocrinology and Metabolism 10261861 1869 https://doi.org/10.1210/jc.2016-3863

M Kawai Y Minabe S Takagai M Ogai H Matsumoto N Mori N Takei 2004 Poor maternal care and high maternal body mass index in pregnancy as a risk factor for schizophrenia in offspring Acta 
Psychiatr Scand 1104257263 https://doi.org/10.1111/j.16000447.2004.00380.x

L Kong G Norstedt M Schalling M Gissler C Lavebratt 2018 The risk of offspring psychiatric disorders in the setting of maternal obesity and diabetes Pediatrics 1423 e20180776 https://doi.org/10.1542/ peds.2018-0776

M Lambert F Ruppelt AK Siem AC Rohenkohl V Kraft D Luedecke M Sengutta R Schröter A Daubmann CU Correll J Gallinat A Karow K Wiedemann D Schöttle 2018 Comorbidity of chronic somatic diseases in patients with psychotic disorders and their influence on 4-year outcomes of integrated care treatment (ACCESS II study) Schizophr Res 193377383 https://doi.org/10.1016/j.schres.2017. 07.036

WA March VM Moore KJ Willson DIW Phillips RJ Norman MJ Davies 2010 The prevalence of polycystic ovary syndrome in a community sample assessed under contrasting diagnostic criteria Hum Reprod 252544551 https://doi.org/10.1093/humrep/dep399

SL Matheson AM Shepherd KR Laurens VJ Carr 2011 A systematic meta-review grading the evidence for non-genetic risk factors and putative antecedents of schizophrenia Schizophr Res 133 1-3 133 142 https://doi.org/10.1016/j.schres.2011.09.020

J Miettunen E Jääskeläinen 2010 Sex differences in wisconsin schizotypy scales-a meta-analysis Schizophr Bull 362347358 https:// doi.org/10.1093/schbul/sbn075

J Miettunen J Veijola M Isohanni T Paunio N Freimer E Jääskeläinen A Taanila J Ekelund MR Järvelin L Peltonen M Joukamaa D Lichtermann 2011 Identifying schizophrenia and other psychoses with psychological scales in the generasl population Journal of Nervous and Mental Disease 1994230238 https://doi.org/10. 1097/NMD.0b013e3182125d2c

NEH Mimouni I Paiva AL Barbotin FE Timzoura D Plassard S Gras Le G Ternier P Pigny S Catteau-Jonard V Simon V Prevot AL Boutillier P Giacobini 2021 Polycystic ovary syndrome is transmitted via a transgenerational epigenetic process Cell Metab 333513 530.e8 https://doi.org/10.1016/j.cmet.2021.01.004

B Misiak D Frydecka O Loska AA Moustafa J Samochowiec J Kasznia B Stańczykiewicz 2018 Testosterone, DHEA and DHEA-S in patients with schizophrenia: a systematic review and meta-analysis Psychoneuroendocrinology 8992102 https://doi.org/10. 1016/j.psyneuen.2018.01.007

B Misiak B Stańczykiewicz Ł Łaczmański D Frydecka 2017 Lipid profile disturbances in antipsychotic-naive patients with first-episode non-affective psychosis: a systematic review and meta-analysis In Schizophrenia Research. 1901827 https://doi.org/10.1016/j. schres.2017.03.031

D Novick W Montgomery T Treuer MV Moneta JM Haro 2016 Sex differences in the course of schizophrenia across diverse regions of the world Neuropsychiatr Dis Treat 1901827 https://doi.org/ 10.2147/NDT.S101151

MME Ollila T Piltonen K Puukka A Ruokonen MR Järvelin JS Tapanainen S Franks L Morin-Papunen 2016 Weight gain and dyslipidemia in early adulthood associate with polycystic ovary syndrome: prospective cohort study J Clin Endocrinol Metab 1012 739747 https://doi.org/10.1210/jc.2015-3543

J Perälä J Suvisaari SI Saarni K Kuoppasalmi E Isometsä S Pirkola T Partonen A Tuulio-Henriksson J Hintikka T Kieseppä T Härkänen S Koskinen J Lönnqvist 2007 Lifetime prevalence of psychotic and bipolar I disorders in a general population Arch Gen Psychiatry $6411928 \mathrm{https}: / /$ doi.org/10.1001/archpsyc.64.1.19

BI Perry R Upthegrove A Thompson S Marwaha S Zammit SP Singh G Khandaker 2019 Dysglycaemia, inflammation and psychosis: findings from the UK ALSPAC birth cohort Schizophr Bull 452 330338 https://doi.org/10.1093/schbul/sby040

TT Piltonen M Ruokojärvi H Karro L Kujanpää L Morin-Papunen JS Tapanainen E Stener-Victorin I Sundrström-Poromaa AL Hirschberg P Ravn D Glintborg JR Mellembakken T Steingrimsdottir M Gibson-Helm E Vanky M Andersen RK Arffman H Teede K Falah-Hassani 2019 Awareness of polycystic ovary syndrome among obstetrician-gynecologists and endocrinologists in Northern Europe PLoS ONE. 1412 e0226074 https://doi.org/10.1371/ journal.pone. 0226074

P Rantakallio 1988 The longitudinal study of the Northern Finland birth cohort of 1966 Paediatr Perinat Epidemiol 215988 https:// doi.org/10.1111/j.1365-3016.1988.tb00180.x

F Rice I Jones A Thapar 2007 The impact of gestational stress and prenatal growth on emotional problems in offspring: a review In Acta Psychiatrica Scandinavica. 1153171183 https://doi.org/10. 1111/j.1600-0447.2006.00895.x

A Riecher-Rössler 2017 Oestrogens, prolactin, hypothalamic-pituitarygonadal axis, and schizophrenic psychoses In the Lancet Psychiatry. 416372 https://doi.org/10.1016/S2215-0366(16)30379-0

A Riecher-Rössler 2002 Oestrogen effects in schizophrenia and their potential therapeutic implications - review Archives of Women's Mental Health. 53111118 https://doi.org/10.1007/ s00737-002-0003-3

Riecher-Rössler A, Rybakowski JK, Pflueger MO, Beyrau R, Kahn RS, Malik P, Fleischhacker WW; EUFEST Study Group (2013) Hyperprolactinemia in antipsychotic-naive patients with firstepisode psychosis. Psychol Med 43(12):2571-2582. https:doi// doi.org/10.1017/S0033291713000226

Risal S, Pei Y, Lu H, Manti M, Fornes R, Pui HP, Zhao Z, Massart J, Ohlsson C, Lindgren E, Crisosto N, Maliqueo M, Echiburú B, Ladrón de Guevara A, Sir-Petermann T, Larsson H, Rosenqvist MA, Cesta C. E, Benrick A, ... Stener-Victorin E. (2019). Prenatal androgen exposure and transgenerational susceptibility to polycystic ovary syndrome. Nature Medicine. 25(12):1894-1904. https:// doi.org/10.1038/s41591-019-0666-1

S Searles JA Makarewicz JA Dumas 2018 The role of estradiol in schizophrenia diagnosis and symptoms in postmenopausal women Schizophrenia Research. 1963538 https://doi.org/10.1016/j. schres.2017.05.024

P Shah Y Iwata F Caravaggio E Plitman EE Brown J Kim N Chan M Hahn G Remington P Gerretsen A Graff-Guerrero 2019 Alterations in body mass index and waist-to-hip ratio in never and minimally treated patients with psychosis: a systematic review and meta-analysis Schizophr Res 208420429 https://doi.org/10. 1016/j.schres.2019.01.005

E Stener-Victorin M Manti R Fornes S Risal H Lu A Benrick 2019 Origins and impact of psychological traits in polycystic ovary syndrome Medical Sciences. 7886 https://doi.org/10.3390/medsc i7080086

S Taponen S Ahonkallio H Martikainen R Koivunen A Ruokonen U Sovio AL Hartikainen A Pouta J Laitinen V King S Franks MI McCarthy MR Järvelin 2004 Prevalence of polycystic ovaries in women with self-reported symptoms of oligomenorrhoea and/or hirsutism: Northern Finland birth cohort 1966 study Hum Reprod 19510831088 https://doi.org/10.1093/humrep/deh214

S Taponen H Martikainen MR Järvelin J Laitinen A Pouta AL Hartikainen U Sovio MI McCarthy S Franks A Ruokonen 2003 Hormonal profile of women with self-reported symptoms of oligomenorrhea and/or hirsutism: Northern Finland birth cohort 1966 study 
J Clin Endocrinol Metab 881141147 https://doi.org/10.1210/ jc.2002-020982

HJ Teede AE Joham E Paul LJ Moran D Loxton D Jolley C Lombard 2013 Longitudinal weight gain in women identified with polycystic ovary syndrome: results of an observational study in young women Obesity 21815261532 https://doi.org/10.1002/oby.20213

HJ Teede ML Misso MF Costello A Dokras J Laven L Moran T Piltonen RJ Norman M Andersen R Azziz A Balen E Baye J Boyle L Brennan F Broekmans P Dabadghao L Devoto D Dewailly L Downes BO Yildiz 2018 Recommendations from the international evidence-based guideline for the assessment and management of polycystic ovary syndrome Human Reproduction. 339 16021618 https://doi.org/10.1093/humrep/dey256

University of Oulu: Northern Finland Birth Cohort 1966. University of Oulu. http://urn.fi/urn:nbn:fi:att:bc1e5408-980e-4a62-b89943bec 3755243

R Upthegrove N Manzanares-Teson NM Barnes 2014 Cytokine function in medication-naive first episode psychosis: a systematic review and meta-analysis Schizophrenia Research. 155 1-3 101 8 https://doi.org/10.1016/j.schres.2014.03.005

M Werf Van Der M Hanssen S Köhler M Verkaaik FR Verhey R Winkel Van J Os Van J Allardyce 2014 Systematic review and collaborative recalculation of 133693 incident cases of schizophrenia Psychol Med 441916 https://doi.org/10.1017/S00332917120027 96

World Health Organization. (2018). Management of physical health conditions in adults with severe mental disorders - WHO Guidelines. World Health Organization. Licence: CC BY-NC-SA 3.0 IGO

Publisher's Note Springer Nature remains neutral with regard to jurisdictional claims in published maps and institutional affiliations. 